\title{
A Selective Culture Medium for Screening Ceftazidime- Avibactam Resistance in Enterobacterales and Pseudomonas aeruginosa
}

\author{
Mustafa Sadek, ${ }^{\mathrm{a}}$ Laurent Poirel,a,b,c Camille Tinguely,a (D) Patrice Nordmann ${ }^{\mathrm{a}, \mathrm{b}, \mathrm{c}, \mathrm{d}}$ \\ aMedical and Molecular Microbiology, Faculty of Science and Medicine, University of Fribourg, Fribourg, Switzerland \\ bINSERM European Unit (IAME), University of Fribourg, Fribourg, Switzerland \\ cSwiss National Reference Center for Emerging Antibiotic Resistance (NARA), University of Fribourg, Fribourg, Switzerland \\ dInstitute for Microbiology, University of Lausanne and University Hospital Centre, Lausanne, Switzerland
}

ABSTRACT The SuperCAZ/AVI medium was developed for screening ceftazidimeavibactam (CZA) resistance among Gram-negative bacteria (Enterobacterales and Pseudomonas aeruginosa). It was evaluated using 50 CZA-susceptible and 42 CZAresistant Gram-negative isolates. Its sensitivity and specificity of detection were $100 \%$. Excellent performance of the medium was also observed by testing spiked stools, with the lower limit of detection ranging from $10^{1}$ to $10^{2} \mathrm{CFU} / \mathrm{ml}$. This screening medium provides the opportunity to detect CZA-resistant isolates regardless of their resistance mechanisms.

KEYWORDS ceftazidime, avibactam, screening, Enterobacterales, Pseudomonas aeruginosa

T he emergence and spread of $\beta$-lactam resistance, especially resistance to carbapenems, are currently of great concern worldwide, particularly in Enterobacterales and Pseudomonas aeruginosa (1). Among the recently developed agents active against multidrug-resistant Gram-negative pathogens, a novel drug combination has been launched, namely, ceftazidime-avibactam (CZA) (2). Avibactam $(\mathrm{AVI})$ is a non- $\beta$-lactam- $\beta$-lactamase inhibitor that inhibits the activities of Ambler class $A$, class $C$, and some class $D \beta$-lactamases, including carbapenemases (e.g., $\operatorname{KPC}, \mathrm{OXA}-48)(3,4)$. However, acquired resistance to CZA is increasingly reported and is mostly related to amino acid substitutions in the active sites of the respective $\beta$-lactamases. Many studies have identified KPC variants in Klebsiella pneumoniae, such as KPC-31, KPC-35, KPC-41, and KPC-50, all conferring resistance to CZA (5-8). Those KPC variants confer acquired resistance to CZA on the corresponding producers mainly as a consequence of decreased inhibitory activity of AVI against those enzymes, but also due to higher hydrolytic efficiency toward ceftazidime (CAZ). In addition, resistance to CZA in Gram-negative bacteria may be related to the production of Ambler class B enzymes (metallo- $\beta$-lactamases [MBL]), such as NDM, VIM, and IMP, or of several non-OXA-48-like class D $\beta$-lactamases, such as OXA-28 or OXA-32, whose hydrolytic activity includes CAZ but which are not inhibited by $A V I$ (1). Furthermore, CZA resistance may be related to overproduction of efflux pumps and/or porin defects (9). Taking into account the increasing use of the CZA combination and consequently the increasing isolation of CZA-resistant Gramnegative bacteria, we have developed a selective culture medium for screening CZA-resistant isolates among Gram-negative species (Enterobacterales, P. aeruginosa).
Citation Sadek M, Poirel L, Tinguely C, Nordmann P. 2020. A selective culture medium for screening ceftazidime-avibactam resistance in Enterobacterales and Pseudomonas aeruginosa. J Clin Microbiol 58:e00965-20. https://doi.org/10.1128/JCM.00965-20. Editor Nathan A. Ledeboer, Medical College of Wisconsin

Copyright $\odot 2020$ American Society for Microbiology. All Rights Reserved.

Address correspondence to Patrice Nordmann, patrice.nordmann@unifr.ch.

Received 1 May 2020

Returned for modification 28 May 2020 Accepted 16 June 2020

Accepted manuscript posted online 24 June 2020

Published 24 August 2020

[This article was published on 24 August 2020 with a CC BY 4.0 copyright line ("Copyright $\odot 2020$ Sadek et al. This is an open-access article distributed under the terms of the Creative Commons Attribution 4.0 International license."). The authors elected to remove open access for the article after publication, necessitating replacement of the original copyright line, and this change was made on 29 September 2020.] 
TABLE 1 Preparation of the SuperCAZ/AVI medium

\begin{tabular}{llll}
\hline Compound & Stock solution ${ }^{a}$ & $\begin{array}{l}\text { Quantity or } \\
\text { vol to add }\end{array}$ & $\begin{array}{l}\text { Final } \\
\text { concn }\end{array}$ \\
\hline CHROMagar Orientation medium & & $13.2 \mathrm{~g}$ & $3.3 \%$ \\
Distilled water & & $400 \mathrm{ml}$ & \\
Ceftazidime pentahydrate & $6 \mathrm{mg} / \mathrm{ml} \mathrm{in} \mathrm{PBS}(\mathrm{pH} 7.2)$ & $400 \mu \mathrm{l}$ & $6 \mu \mathrm{g} / \mathrm{ml}$ \\
Avibactam sodium hydrate & $4 \mathrm{mg} / \mathrm{ml} \mathrm{in} \mathrm{water}$ & $400 \mu \mathrm{l}$ & $4 \mu \mathrm{g} / \mathrm{ml}$ \\
$\mathrm{ZnSO}_{4} \cdot 7 \mathrm{H}_{2} \mathrm{O}$ & $70 \mathrm{mg} / \mathrm{ml}$ in water & $400 \mu \mathrm{l}$ & $70 \mu \mathrm{g} / \mathrm{ml}$ \\
Daptomycin $_{\text {Amphotericin B }}$ & $10 \mathrm{mg} / \mathrm{ml}$ in water & $400 \mu \mathrm{l}$ & $10 \mu \mathrm{g} / \mathrm{ml}$ \\
\hline
\end{tabular}

aPBS, phosphate-buffered saline.

${ }^{b}$ The volume of $400 \mathrm{ml}$ of SuperCAZ/AVI medium was for 20 plates.

\section{MATERIALS AND METHODS}

The SuperCAZ/AVI medium. In the design of our medium (named the SuperCAZ/AVI medium), the necessity of preventing contamination by Gram-positive bacteria and fungi was considered. Based on our experience in the development of screening media (10), the optimal screening medium was based on the CHROMagar Orientation medium (reference RT412; CHROMagar, Paris, France), which is commonly used as a differential medium for the isolation and differentiation of common urinary tract pathogens. The CZA resistance breakpoint is defined as $>8 \mu \mathrm{g} / \mathrm{ml}$ for Enterobacterales and $P$. aeruginosa with a fixed concentration of AVI (4 $\mu \mathrm{g} / \mathrm{ml})$ (11). The optimal final concentration of CAZ was $6 \mu \mathrm{g} / \mathrm{ml}$ with a fixed concentration of AVI at $4 \mu \mathrm{g} / \mathrm{ml}$. Since Gram-positive bacteria, such as Enterococcus, Streptococcus, and Staphylococcus strains, may grow on CHROMagar Orientation medium, daptomycin (code 461375000; Acros Organics) (which can be replaced by vancomycin) was added as an anti-Gram-positive molecule at a final concentration of $10 \mu \mathrm{g} / \mathrm{ml}$. Amphotericin B (code 45590050; Acros Organics) was also added as an antifungal at a final concentration of $5 \mu \mathrm{g} / \mathrm{ml}$. In addition, $\mathrm{ZnSO}_{4}(70 \mu \mathrm{g} / \mathrm{ml})$ was added to enhance the activity of $\mathrm{MBL}$ producers (10). The stock solutions of $\mathrm{CAZ}, \mathrm{AVI}$, daptomycin, and amphotericin $\mathrm{B}$ were prepared as shown in Table 1 and may be kept at $-20^{\circ} \mathrm{C}$ for 1 year. For the preparation of the SuperCAZ/AVI medium, the diluted powder of CHROMagar Orientation medium was autoclaved at $121^{\circ} \mathrm{C}$ for $15 \mathrm{~min}$. After the medium was cooled for $1 \mathrm{~h}$ at $56^{\circ} \mathrm{C}$, the antibiotic stock solutions were added (Table 1). The SuperCAZ/AVI plates were stored at $4^{\circ} \mathrm{C}$ and were protected from direct light exposure before use, for as long as 1 week.

Susceptibility testing. The MICs of CZA were determined using Etest strips (bioMérieux, La Balmeles-Grottes, France) on Mueller-Hinton agar plates at $37^{\circ} \mathrm{C}$, and the results were interpreted according to the latest EUCAST breakpoints for Enterobacterales and $P$. aeruginosa (i.e., susceptibility [S], $\leq 8 \mu \mathrm{g} / \mathrm{ml}$; resistance $[\mathrm{R}],>8 \mu \mathrm{g} / \mathrm{ml}$ ) (Table 2) (10).

\section{RESULTS}

A total of 92 isolates of worldwide origin were included in this study to evaluate the performance of the SuperCAZ/AVI medium. The $\beta$-lactamase contents of all strains were characterized at the molecular level by PCR and sequencing or, for some isolates, by whole-genome sequencing (Table 2). A total of 50 strains were susceptible to CZA (40 Enterobacterales, including Enterobacter cloacae, K. pneumoniae, and Escherichia coli, and 10 P. aeruginosa strains), and 42 were resistant to CZA (20 Enterobacterales, including E. cloacae, K. pneumoniae, and E. coli, and 22 P. aeruginosa strains) (Table 2).

Starting with an optical density of a 0.5 McFarland standard (an inoculum of $\sim 1.5 \times 10^{8} \mathrm{CFU} / \mathrm{ml}$ ), serial 10 -fold dilutions were made in $0.85 \%$ saline solution, and $100-\mu \mathrm{l}$ aliquots of each dilution were plated onto the SuperCAZ/AVI medium. To quantify the viable bacteria in each dilution step, tryptic soy agar plates were inoculated concomitantly with $100 \mu \mathrm{l}$ of each suspension and were incubated overnight at $37^{\circ} \mathrm{C}$. Viable colonies were counted the following day. When no growth was observed after $18 \mathrm{~h}$, incubation was extended up to $48 \mathrm{~h}$ in order to definitely assess the negativity of the culture. The lower limit of detection for the strains tested was determined using the SuperCAZ/AVI medium.

The sensitivity and specificity cutoff values for the detection of CZA-resistant Enterobacterales and $P$. aeruginosa were set at $1 \times 10^{3} \mathrm{CFU} / \mathrm{ml}$, i.e., the CZA-resistant isolates recovered on SuperCAZ/AVI medium plates at $<1 \times 10^{3} \mathrm{CFU} / \mathrm{ml}$ were considered positive, while the CZA-susceptible isolates grown using an inoculum of $\geq 1 \times 10^{3}$ $\mathrm{CFU} / \mathrm{ml}$ were considered negative (10). All the CZA-resistant isolates could be recovered within $24 \mathrm{~h}$ on SuperCAZ/AVI medium plates by using an inoculum below the cutoff value of $1 \times 10^{3} \mathrm{CFU} / \mathrm{ml}\left(1 \times 10^{1}\right.$ to $\left.1 \times 10^{2} \mathrm{CFU} / \mathrm{ml}\right)$ (Table 2). In contrast, growth of 
TABLE 2 MICS of CZA for the strains tested and detection limits of the SuperCAZ/AVI screening medium

\begin{tabular}{|c|c|c|c|c|c|c|c|}
\hline \multirow[b]{2}{*}{ Category and strain } & \multirow[b]{2}{*}{ Species } & \multirow[b]{2}{*}{ Origin } & \multirow{2}{*}{$\begin{array}{l}\text { MIC of CZAa } \\
\text { (mg/liter) }\end{array}$} & \multirow{2}{*}{$\begin{array}{l}\text { CZA } \\
\text { susceptibility } \\
\text { or resistance }^{b}\end{array}$} & \multirow{2}{*}{$\begin{array}{l}\text { Resistance } \\
\text { determinant(s) }\end{array}$} & \multicolumn{2}{|c|}{$\begin{array}{l}\text { Lower limit of } \\
\text { detection (CFU/ } \\
\mathrm{ml})^{c} \text { in: }\end{array}$} \\
\hline & & & & & & Culture & Stoolsc \\
\hline \multicolumn{8}{|l|}{ Enterobacterales } \\
\hline $\mathrm{R} 1433$ & Enterobacter cloacae & France & 0.19 & S & Wild type & $\geq 10^{8}$ & $\geq 10^{7}$ \\
\hline $\mathrm{R} 254$ & Klebsiella pneumoniae & France & 0.064 & $S$ & $\begin{array}{l}\text { Porin deficiency, SHV, } \\
\text { AmpC }\end{array}$ & $\underline{10^{6}}$ & $\underline{10^{6}}$ \\
\hline $\mathrm{R} 2077$ & Escherichia coli & Switzerland & 0.5 & S & ACC-1 & $\overline{10^{7}}$ & $\underline{10^{7}}$ \\
\hline R1291 & Escherichia coli & USA & 0.032 & S & OXA-1 & $\overline{\geq 10^{8}}$ & $\overline{\geq 10^{7}}$ \\
\hline R1335 & Escherichia coli & France & 0.064 & S & TEM-1 & $\overline{10^{8}}$ & $\overline{\geq 10^{7}}$ \\
\hline R941 & Enterobacter cloacae & Switzerland & 1.5 & S & TEM-1 & $\overline{10^{7}}$ & $\overline{10^{7}}$ \\
\hline R1906 & Escherichia coli & France & 0.75 & S & SHV-12 & $\overline{>10^{8}}$ & $\overline{>10^{7}}$ \\
\hline R2180 & Enterobacter cloacae & France & 2 & S & GES-5 & $\overline{>10^{8}}$ & $>10^{7}$ \\
\hline R1039 & Escherichia coli & Vietnam & 0.25 & S & VEB-1, OXA-10, TEM-1 & $\overline{>10^{8}}$ & $\overline{\geq 10^{7}}$ \\
\hline R1104 & Klebsiella pneumoniae & Thailand & 0.75 & S & VEB-1 & $\overline{>10^{8}}$ & $\overline{>10^{7}}$ \\
\hline $\mathrm{R} 1103$ & Klebsiella pneumoniae & Thailand & 0.5 & S & VEB-1 & $>10^{7}$ & $>10^{7}$ \\
\hline $\mathrm{R} 144$ & Escherichia coli & France & 0.75 & S & VEB-1 & $\geq 10^{8}$ & $\geq 10^{7}$ \\
\hline R1105 & Klebsiella pneumoniae & Thailand & 0.25 & S & VEB-1 & $\overline{>10^{8}}$ & $\overline{>10^{8}}$ \\
\hline R2658 & Escherichia coli & France & 0.125 & S & VEB-1, TEM-1, OXA-10 & $\geq 10^{8}$ & $\geq 10^{7}$ \\
\hline R3659 & Escherichia coli & USA & 0.5 & S & $\begin{array}{l}\text { KPC-2 (E. coli DH10B/ } \\
\left.\text { pBR322 bla } a_{\mathrm{KPC}-2}\right)\end{array}$ & $\geq 10^{8}$ & $\overline{\geq 10^{8}}$ \\
\hline R99 & Klebsiella pneumoniae & France & 0.5 & $S$ & $\mathrm{KPC}-2$ & $\underline{10^{7}}$ & $\underline{10^{5}}$ \\
\hline R3521 & Klebsiella pneumoniae & Switzerland & 1.5 & $S$ & $\mathrm{KPC}-2$ & $\overline{10^{5}}$ & $\underline{10^{6}}$ \\
\hline R3668 & Escherichia coli & USA & 0.064 & $S$ & $\begin{array}{l}\mathrm{KPC}-2[\text { E. coli } \mathrm{DH} 10 \mathrm{~B} / \\
\left.\mathrm{pBC} \mathrm{SK}(+) b l a_{\mathrm{KPC}-2}\right]\end{array}$ & $\overline{\geq 10^{8}}$ & $\geq 10^{8}$ \\
\hline R82 & Escherichia coli & France & 0.047 & S & KPC-2 & $\geq 10^{8}$ & $\geq 10^{7}$ \\
\hline R22 & Escherichia coli & France & 0.094 & $S$ & OXA-48 & $>10^{8}$ & $>10^{7}$ \\
\hline R740 & Escherichia coli & The Netherlands & 1 & $S$ & OXA-48 & $\overline{>10^{8}}$ & $>10^{7}$ \\
\hline R19 & Klebsiella pneumoniae & France & 0.5 & $S$ & OXA-48 & $10^{7}$ & $10^{6}$ \\
\hline R23 & Klebsiella pneumoniae & France & 0.5 & $S$ & OXA-48 & $10^{7}$ & $10^{7}$ \\
\hline N59 & Escherichia coli & Switzerland & 0.023 & $S$ & OXA-181 & $>10^{8}$ & $>10^{7}$ \\
\hline R131 & Klebsiella pneumoniae & France & 1.5 & $\mathrm{~S}$ & OXA-181 & $10^{8}$ & $>10^{7}$ \\
\hline R3338 & Klebsiella pneumoniae & USA & 24 & $\mathrm{R}$ & CMY-4, VIM-1 & $\overline{10^{2}}$ & $10^{1}$ \\
\hline R169 & Klebsiella pneumoniae & USA & 24 & $\mathrm{R}$ & VIM-19 & $10^{1}$ & $10^{1}$ \\
\hline N284 & Enterobacter cloacae & Switzerland & 48 & $\mathrm{R}$ & VIM-1 & $10^{1}$ & $10^{1}$ \\
\hline R48 & Klebsiella pneumoniae & France & $>256$ & $\mathrm{R}$ & VIM-1 & $10^{1}$ & $10^{1}$ \\
\hline R61 & Escherichia coli & France & 24 & $\mathrm{R}$ & VIM-1, SHV-12 & $10^{2}$ & $10^{1}$ \\
\hline R63 & Klebsiella pneumoniae & France & 24 & $\mathrm{R}$ & VIM-19 & $10^{1}$ & $10^{1}$ \\
\hline N6 & Escherichia coli & Switzerland & $>256$ & $\mathrm{R}$ & NDM-5 & $10^{2}$ & $10^{2}$ \\
\hline R464 & Escherichia coli & France & $>256$ & $\mathrm{R}$ & NDM-4, OXA-1 & $10^{1}$ & $10^{1}$ \\
\hline R466 & Escherichia coli & France & $>256$ & $\mathrm{R}$ & NDM-4, OXA-1, CTX-M-15 & $10^{2}$ & $10^{1}$ \\
\hline R3778 & Klebsiella pneumoniae & Spain & 48 & $\mathrm{R}$ & KPC-3 D179Y & $10^{1}$ & $10^{1}$ \\
\hline R3780 & Klebsiella pneumoniae & Spain & $>256$ & $\mathrm{R}$ & KPC-3 G168N E169H & $10^{1}$ & $10^{1}$ \\
\hline R3781 & Klebsiella pneumoniae & Spain & 64 & $\mathrm{R}$ & KPC-3 E169P L172T & $10^{1}$ & $10^{1}$ \\
\hline R3776 & Klebsiella pneumoniae & Spain & 96 & $\mathrm{R}$ & KPC-3 D179Y & $10^{1}$ & $10^{1}$ \\
\hline R3777 & Klebsiella pneumoniae & Spain & $>256$ & $\mathrm{R}$ & KPC-3 D179Y A172T & $10^{1}$ & $10^{1}$ \\
\hline N435 & Klebsiella pneumoniae & Switzerland & $>256$ & $\mathrm{R}$ & KPC-41 & $10^{1}$ & $10^{1}$ \\
\hline N859 & Klebsiella pneumoniae & Switzerland & $>256$ & $\mathrm{R}$ & KPC-50 & $10^{1}$ & $10^{1}$ \\
\hline
\end{tabular}


TABLE 2 (Continued)

\begin{tabular}{|c|c|c|c|c|c|c|c|}
\hline \multirow[b]{2}{*}{ Category and strain } & \multirow[b]{2}{*}{ Species } & \multirow[b]{2}{*}{ Origin } & \multirow{2}{*}{$\begin{array}{l}\text { MIC of CZAa } \\
\text { (mg/liter) }\end{array}$} & \multirow{2}{*}{$\begin{array}{l}\text { CZA } \\
\text { susceptibility } \\
\text { or resistance }^{b}\end{array}$} & \multirow{2}{*}{$\begin{array}{l}\text { Resistance } \\
\text { determinant(s) }\end{array}$} & \multicolumn{2}{|c|}{$\begin{array}{l}\text { Lower limit of } \\
\text { detection (CFU/ } \\
\mathrm{ml})^{c} \text { in: }\end{array}$} \\
\hline & & & & & & Culture & Stoolsc \\
\hline R3671 & Escherichia coli & USA & $>128$ & $\mathrm{R}$ & $\begin{array}{l}\text { KPC-2 (E. coli DH10B/ } \\
\text { pBR322 KPC-2 D179M) }\end{array}$ & $10^{2}$ & $10^{2}$ \\
\hline R3779 & Klebsiella pneumoniae & Spain & 128 & $\mathrm{R}$ & KPC-3 D179Y & $10^{2}$ & $10^{2}$ \\
\hline $\mathrm{R} 72$ & Escherichia coli & France & 128 & $\mathrm{R}$ & IMP-1 & $10^{1}$ & $10^{1}$ \\
\hline $\mathrm{R} 73$ & Klebsiella pneumoniae & France & $>256$ & $\mathrm{R}$ & IMP-1 & $10^{1}$ & $10^{1}$ \\
\hline \multicolumn{8}{|c|}{ Pseudomonas aeruginosa } \\
\hline R1553 & Pseudomonas aeruginosa & France & 1.5 & $\mathrm{~S}$ & None (wild type) & $\geq 10^{8}$ & $10^{7}$ \\
\hline R2267 & Pseudomonas aeruginosa & France & 0.75 & $\mathrm{~S}$ & None (wild type) & $\geq 10^{8}$ & $10^{7}$ \\
\hline N382 & Pseudomonas aeruginosa & Switzerland & 0.38 & $\mathrm{~S}$ & None (wild type) & $\overline{10^{6}}$ & $\overline{10^{7}}$ \\
\hline N339 & Pseudomonas aeruginosa & Switzerland & 0.5 & $\mathrm{~S}$ & None (wild type) & $\geq 10^{8}$ & $10^{7}$ \\
\hline N146 & Pseudomonas aeruginosa & Switzerland & 4 & $\mathrm{~S}$ & GES-5 & $10^{4}$ & $\overline{10^{6}}$ \\
\hline N254 & Pseudomonas aeruginosa & Switzerland & 1 & $\mathrm{~S}$ & None (wild type) & $\geq 10^{8}$ & $\overline{10^{8}}$ \\
\hline N214 & Pseudomonas aeruginosa & Switzerland & 0.5 & $\mathrm{~S}$ & None (wild type) & $>10^{8}$ & $\overline{10^{7}}$ \\
\hline R1187 & Pseudomonas aeruginosa & Belgium & 4 & $\mathrm{~S}$ & BEL-2 & $\underline{10^{6}}$ & $\underline{10^{5}}$ \\
\hline R1188 & Pseudomonas aeruginosa & Brazil & 2 & $S$ & CTX-M-2 & $\geq 10^{8}$ & $\geq 10^{7}$ \\
\hline R3451 & Pseudomonas aeruginosa & France & 1 & $\mathrm{~S}$ & GES-6 & $\underline{10^{6}}$ & $\underline{10^{7}}$ \\
\hline R3680 & Pseudomonas aeruginosa & USA & 24 & $\mathrm{R}$ & Unknown mechanism & $\overline{10^{1}}$ & $\overline{10^{2}}$ \\
\hline R3681 & Pseudomonas aeruginosa & USA & 32 & $\mathrm{R}$ & Unknown mechanism & $10^{1}$ & $10^{1}$ \\
\hline R3682 & Pseudomonas aeruginosa & USA & 64 & $\mathrm{R}$ & Unknown mechanism & $10^{1}$ & $10^{1}$ \\
\hline R3683 & Pseudomonas aeruginosa & USA & $>256$ & $\mathrm{R}$ & Unknown mechanism & $10^{1}$ & $10^{1}$ \\
\hline R1308 & Pseudomonas aeruginosa & France & $>256$ & $\mathrm{R}$ & OXA-28 & $10^{1}$ & $10^{2}$ \\
\hline R1311 & Pseudomonas aeruginosa & France & 12 & $\mathrm{R}$ & OXA-32 & $10^{1}$ & $10^{1}$ \\
\hline R609 & Pseudomonas aeruginosa & Turkey & 64 & $\mathrm{R}$ & VIM-2 & $10^{1}$ & $10^{1}$ \\
\hline R50 & Pseudomonas aeruginosa & France & 24 & $\mathrm{R}$ & VIM-2 & $10^{2}$ & $10^{1}$ \\
\hline R51 & Pseudomonas aeruginosa & France & $>256$ & $\mathrm{R}$ & VIM-2 & $10^{1}$ & $10^{1}$ \\
\hline $\mathrm{R} 52$ & Pseudomonas aeruginosa & France & 16 & $\mathrm{R}$ & VIM-2 & $10^{1}$ & $10^{1}$ \\
\hline R54 & Pseudomonas aeruginosa & France & $>256$ & $\mathrm{R}$ & VIM-2 & $10^{2}$ & $10^{1}$ \\
\hline R598 & Pseudomonas aeruginosa & France & 24 & $\mathrm{R}$ & VIM-2 & $10^{1}$ & $10^{1}$ \\
\hline R599 & Pseudomonas aeruginosa & France & 16 & $\mathrm{R}$ & VIM-2 & $10^{1}$ & $10^{1}$ \\
\hline R600 & Pseudomonas aeruginosa & Japan & 16 & $\mathrm{R}$ & VIM-2 & $10^{2}$ & $10^{1}$ \\
\hline R604 & Pseudomonas aeruginosa & The Netherlands & 12 & $\mathrm{R}$ & VIM-2 & $10^{1}$ & $10^{1}$ \\
\hline R608 & Pseudomonas aeruginosa & France & 16 & $\mathrm{R}$ & VIM-2 & $10^{2}$ & $10^{2}$ \\
\hline R610 & Pseudomonas aeruginosa & France & 32 & $\mathrm{R}$ & VIM-2 & $10^{2}$ & $10^{2}$ \\
\hline N885 & Pseudomonas aeruginosa & Switzerland & $>256$ & $\mathrm{R}$ & NDM-1 & $10^{1}$ & $10^{1}$ \\
\hline N520 & Pseudomonas aeruginosa & Switzerland & $>256$ & $\mathrm{R}$ & NDM-1 & $10^{1}$ & $10^{1}$ \\
\hline N521 & Pseudomonas aeruginosa & Switzerland & $>256$ & $\mathrm{R}$ & NDM-1 & $10^{1}$ & $10^{1}$ \\
\hline R186 & Pseudomonas aeruginosa & France & 16 & $\mathrm{R}$ & NDM-6 & $10^{2}$ & $10^{2}$ \\
\hline R2760 & Pseudomonas aeruginosa & France & $>256$ & $\mathrm{R}$ & NDM-1 & $10^{2}$ & $10^{1}$ \\
\hline
\end{tabular}

${ }^{a}$ CZA, ceftazidime-avibactam. MICs of CZA were determined using Etest.

${ }^{b} \mathrm{R}$, resistant; S, susceptible.

cUnderlined CFU counts are considered negative results (cutoff values were set at $>10^{3} \mathrm{CFU} / \mathrm{ml}$ ).

the CZA-susceptible isolates was possible only when an inoculum of $>10^{3} \mathrm{CFU} / \mathrm{ml}$ was used (the lower limit of detection was above the cutoff value of $10^{3} \mathrm{CFU} / \mathrm{ml}$ ), giving rise to $100 \%$ sensitivity and specificity.

Spiked stools were also tested with the same representative collection of CZAresistant and -susceptible Gram-negative bacteria $(n=92)$ using this selective culture medium. Spiked fecal samples were made by adding $100 \mu \mathrm{l}$ of serial 10-fold bacterial dilutions to $900 \mu \mathrm{l}$ of a stool suspension. Stool suspensions were obtained by suspending $6 \mathrm{~g}$ of freshly pooled feces from healthy volunteers in $60 \mathrm{ml}$ of distilled water as described previously (10). Aliquots $(100 \mu \mathrm{l})$ of the spiked stool suspension were inoculated onto the SuperCAZ/AVI medium. Aliquots (100 $\mu \mathrm{l})$ of stool suspensions with no bacteria added were plated onto the SuperCAZ/AVI medium as negative controls. The lower limit of detection was below the cutoff value for all CZA-resistant strains with which stools were spiked, ranging from $10^{1}$ to $10^{2} \mathrm{CFU} / \mathrm{ml}$, whereas the lower limit of detection for the CZA-susceptible strains was above the cutoff value, at $\geq 10^{6} \mathrm{CFU} / \mathrm{ml}$ (Table 2). Sensitivity and specificity were determined using the same cutoff value, set 
at $10^{3} \mathrm{CFU} / \mathrm{ml}$ (10). Again, the sensitivity and specificity of the SuperCAZ/AVI medium for isolating CZA-resistant isolates were both $100 \%$.

To assess the storage stability of the SuperCAZ/AVI medium, Candida albicans and Staphylococcus aureus strains, as well as the CZA-susceptible E. coli ATCC 25955 reference strain, were subcultured daily onto the SuperCAZ/AVI medium from a single batch of medium stored at $4^{\circ} \mathrm{C}$. No growth was observed consistently for at least a 7-day period.

\section{DISCUSSION}

The SuperCAZ/AVI medium constitutes an adequate screening medium for the detection of CZA-resistant bacteria regardless of their resistance mechanisms. This SuperCAZ/AVI medium may be used for the screening of patients potentially colonized with CZA-resistant strains in order to rapidly implement infection control measures aimed at limiting their spread. This medium is also adequate for epidemiological surveys aiming to evaluate the prevalence of CZA-resistant Gram-negative bacteria in a given population. Further clinical evaluation of the proposed medium in daily clinical practice is needed. It may be useful for rapid identification of outbreaks of CZA-resistant strains, such as those reported in the United States (12) and Italy (13).

\section{ACKNOWLEDGMENTS}

We thank Luis Martinez-Martinez (Spain) and Robert Bonomo (United States) for the gifts of several CZA-resistant strains.

This work was funded by the Swiss National Science Foundation (projects FNS407240_177381 and FNS-407240_177382).

\section{REFERENCES}

1. Nordmann P, Poirel L. 2019. Epidemiology and diagnostics of carbapenem resistance in Gram negative bacteria. Clin Infect Dis 69(Suppl 7):S521-S528. https://doi.org/10.1093/cid/ciz824.

2. Wright $\mathrm{H}$, Bonomo RA, Paterson DL. 2017. New agents for the treatment of infections with Gram-negative bacteria: restoring the miracle or false dawn? Clin Microbiol Infect 23:704-712. https://doi.org/10.1016/j.cmi 2017.09.001.

3. Zhanel GG, Lawson CD, Adam H, Schweizer F, Zelenitsky S, Lagacé-Wiens PRS, Denisuik A, Rubinstein E, Gin AS, Hoban DJ, Lynch JP, Karlowsky JA. 2013. Ceftazidime-avibactam: a novel cephalosporin/ $\beta$-lactamase inhibitor combination. Drugs 73:159-177. https://doi.org/10.1007/s40265 -013-0013-7.

4. Sharma R, Park TE, Moy S. 2016. Ceftazidime-avibactam: a novel cephalosporin/ $\beta$-lactamase inhibitor combination for the treatment of resistant gram-negative organisms. Clin Ther 38:431-444. https://doi .org/10.1016/j.clinthera.2016.01.018.

5. Barnes MD, Winkler ML, Taracila MA, Page MG, Desarbre E, Kreiswirth BN Shields RK, Nguyen M-H, Clancy C, Spellberg B, Papp-Wallace KM, Bonomo RA. 2017. Klebsiella pneumoniae carbapenemase-2 (KPC-2), substitutions at Ambler position Asp179, and resistance to ceftazidime-avibactam: unique antibiotic-resistant phenotypes emerge from $\beta$-lactamase protein engineering. mBio 8:e00528-17. https://doi.org/10.1128/mBio.00528-17.

6. Mueller L, Masseron A, Prod'Hom G, Galperine T, Greub G, Poirel L, Nordmann P. 2019. Phenotypic, biochemical, and genetic analysis of KPC-41, a KPC-3 variant conferring resistance to ceftazidime-avibactam and exhibiting reduced carbapenemase activity. Antimicrob Agents Chemother 63:e01111-19. https://doi.org/10.1128/AAC.01111-19.

7. Poirel L, Vuillemin X, Juhas M, Masseron A, Bechtel-Grosch U, Tiziani S,
Mancini S, Nordmann P. 26 May 2020. KPC-50 confers resistance to ceftazidime-avibactam associated with reduced carbapenemase activity. Antimicrob Agents Chemother https://doi.org/10.1128/AAC.00321-20.

8. Hemarajata P, Humphries RM. 2019. Ceftazidime/avibactam resistance associated with L169P mutation in the omega loop of KPC-2. J Antimicrob Chemother 74:1241-1243. https://doi.org/10.1093/jac/dkz026.

9. Wang Y, Wang J, Wang R, Cai Y. 19 December 2019. Resistance to ceftazidime-avibactam and underlying mechanisms. J Glob Antimicrob Resist 22:18-27. https://doi.org/10.1016/j.jgar.2019.12.009.

10. Nordmann P, Girlich D, Poirel L. 2012. Detection of carbapenemase producers in Enterobacteriaceae by use of a novel screening medium. J Clin Microbiol 50:2761-2766. https://doi.org/10.1128/JCM.06477-11.

11. EUCAST. 2020. Breakpoint tables for interpretation of MICs and zone diameters, version 10.0. https://www.eucast.org/fileadmin/src/media/PDFs/ EUCAST_files/Breakpoint_tables/v_10.0_Breakpoint_Tables.pdf.

12. Aitken $S L$, Tarrand JJ, Deshpande $L M$, Tverdek FP, Jones $A L$, Shelburne SA, Prince RA, Bhatti MM, Rolston KVI, Jones RN, Castanheira M, Chemaly RF. 2016. High rates of non-susceptibility to ceftazidime-avibactam and identification of New Delhi metallo- $\beta$-lactamase production in Enterobacteriaceae bloodstream infections at a major cancer center. Clin Infect Dis 63:954-958. https://doi.org/10.1093/cid/ciw398.

13. Giani T, Antonelli A, Sennati S, Di Pilato V, Chiarelli A, Cannatelli A, Gatsch C, Luzzaro F, Spanu T, Stefani S, Rossolini GM. 2020. Results of the Italian infection-Carbapenem Resistance Evaluation Surveillance Trial (iCREST-IT): activity of ceftazidime/avibactam against Enterobacterales isolated from urine. J Antimicrob Chemother 75:979-983. https://doi.org/10.1093/jac/ dkz547. 